Ma et al. suggest that hypertension and hyperglycemia impair vascular function and lead to the development of both ED and CHD. They recommend that ED should be considered as an early marker of CHD in patients with type 2 diabetes and that erectile functional status should be recorded and included in assessments of cardiovascular risk.

Original article Ma RC et al. (2008) Erectile dysfunction predicts coronary heart disease in type 2 diabetes. J Am Coll Cardiol 51: 2045-2050

\section{Rimonabant has limited effects on atherosclerosis}

Abdominal adiposity and the metabolic syndrome are associated with an increased risk of atherosclerotic cardiovascular disease. Inhibition of cannabinoid type 1 (CB1) receptors suppresses appetite and can ameliorate some features of the metabolic syndrome that are associated with abdominal obesity (dyslipidemia, inflammatory markers, glycemia). Nissen and colleagues investigated whether treatment with one such CB1 antagonist, rimonabant, could slow the progression of coronary atherosclerosis in obese patients with the metabolic syndrome.

The STRADIVARIUS multicenter trial enrolled patients (average age <60 years) with preexisting coronary disease and abdominal obesity and those who met criteria for the metabolic syndrome or were current smokers. Patients received $20 \mathrm{mg}$ rimonabant or placebo daily for 18-20 months, together with dietary advice to achieve a moderately hypocaloric diet. A total of 676 patients underwent intravascular ultrasonography of the coronary arteries at both baseline and at the end of follow-up.

Weight loss and reduction in waist circumference were greater in patients treated with rimonabant than those receiving placebo. Rimonabant treatment elevated HDL cholesterol levels by $22.4 \%$ and reduced triglyceride levels by $20.5 \%$, demonstrating the capacity of the CB1 antagonist to reverse some effects of the metabolic syndrome. Rimonabant treatment did not significantly slow the rate of progression of atherosclerosis; however, this therapy did reduce patients' total atheroma volume. Rimonabant was also associated with adverse psychiatric events.

Although the authors expected a greater reduction in the progression of atherosclerosis, they believe that managing coronary disease by treatment of obesity using CB1 inhibition warrants further study.

Original article Nissen SE et al. (2008) Effect of rimonabant on progression of atherosclerosis in patients with abdominal obesity and coronary artery disease: the STRADIVARIUS randomized controlled trial. JAMA 299: $1547-1560$

\section{Pioglitazone inhibits progression of atherosclerosis}

People with type 2 diabetes are particularly susceptible to coronary artery disease; however, whether glucose-lowering medications affect the progression of atherosclerosis in such individuals is uncertain. Results of the recent PERISCOPE trial suggest that the thiazolidinedione compound pioglitazone, which increases the sensitivity of peripheral tissues to insulin, slows the progression of atherosclerosis.

Nissen et al. assessed 543 patients with type 2 diabetes and coronary artery disease who were randomly assigned to receive either pioglitazone or glimeparide, an insulin secretagogue. Participants underwent coronary intravascular ultrasonography examination at baseline and after 18 months of treatment.

Mean atheroma volume increased by $0.73 \%$ (95\% Cl $0.33 \%-1.12 \%)$ in the glimeparide group, whereas volume decreased by $0.16 \%$ (95\% Cl $-0.57 \%$ to $0.25 \%$ ) in the pioglitazone group $(P=0.002)$. Pioglitazone also had morefavorable effects on $\mathrm{HbA}_{1 \mathrm{c}}$, fasting blood glucose, HDL cholesterol, triglyceride and C-reactive protein levels than did glimeparide, but was associated with greater mean weight gain. The incidence of major cardiovascular events was similar in the two treatment groups. Glimeparide was associated with higher incidences of angina and hypoglycemia, whereas pioglitazone was associated with higher incidences of peripheral edema and fracture.

The mechanism by which pioglitazone inhibits atherogenesis remains to be determined; however, the favorable effects on atherosclerosis-related biomarkers observed for pioglitazone have not been seen with other thiazolidinediones.

Original article Nissen SE et al. (2008) Comparison of pioglitazone vs glimepiride on progression of coronary atherosclerosis in patients with type 2 diabetes: the PERISCOPE randomized controlled trial. JAMA 299: $1561-1573$ 